\title{
Physical Properties of Twisted Structures. II. Industrial Yarns, Cords, and Ropes
}

\author{
NING PAN, ${ }^{1}$ DAVID BROOKSTEIN ${ }^{2}$ \\ ${ }^{1}$ Division of Textiles and Clothing, Biological and Agricultural Engineering Department, University of California, \\ Davis, California 95616 \\ ${ }^{2}$ Philadelphia College of Textile \& Science, Philadelphia, Pennsylvania 19144
}

Received 8 November 2000; accepted 18 November 2000

\begin{abstract}
In this article, we review the progress made in the area of industrial twisted fibrous structures. First, we focus on the general geometrical description of the structures, and then move to issues including the tensile behavior and fracture process. The effects of fiber bending and torsion, strain rate, and viscoelasticity are also discussed. The influences of both dynamic and fatigue loadings are studied as well. Discussion on the fiber blending and on the behavior of blended hybrid structures is provided. Further, a special section is included in which six specific topical areas, their significance toward textile science, and the progress made in these areas are introduced and summarized. Finally, experimental issues are discussed. Although this article is intended to serve as a complete review of the subject, because of the limit of both time and space, we can only focus on the areas in which we are most familiar. This article, therefore, is by no means exhaustive or authoritative in every topic discussed. () 2002 John Wiley \& Sons, Inc. J Appl Polym Sci 83: 610-630, 2002
\end{abstract}

Key words: twisted fibrous structures; mechanics of ropes; constitutive equations; hybrid cases; some fundamental issues

\section{INTRODUCTION}

Industrial (or technical) textiles are defined as "textile materials and products intended for enduses other than clothing, household, furnishing and floorcovering, where the fabric or fibrous component is selected principally (but not exclusively) for its performance and properties as opposed to its aesthetic or decorative characteristics." Industrial textiles may thus include those applied in agricultural areas such as gardening and landscaping, forestry, and animal husbandry; in building or construction areas such as membranes, materials for

Correspondence to: N. Pan.

Journal of Applied Polymer Science, Vol. 83, 610-630 (2002)

() 2002 John Wiley \& Sons, Inc. lightweight and solid constructions; in geotech areas for road and dam construction, soil sealing, and drainage systems; in other industrial areas for filtration, sealers, and insulation applications; in the medical area for hygiene and protection purposes; and in the mobile industry and sports areas. ${ }^{2}$ The scope of this article will only deal with the industrial twisted fibrous structures which include yarns, cords, and ropes.

The main advantages of using twisted fibrous structures are for their strength, weight, flexibility, and elastic behavior; with regard to the latter, "when flax is twisted to form a cord, it exhibits maximum axial stiffness and minimum bending stiffness." 3

The major differences between industrial yarns, cords, and ropes, and yarns for clothing 
purposes lies in the requirement in durability, load-carrying capacity, and other physical, chemical, or service-related properties. Another distinction is that the majority of modern industrial yarns are often made of filament instead of staple fibers.

The mechanics of twisted fibrous structures is such a classic topic in textile science that there have been several books or monographs devoted to it. ${ }^{4-8}$ It is therefore not our purpose to provide all the detailed treatment on related topics. Rather, we list the major important issues and contributors in the area, the current status of the fields, and more importantly, stress, whenever possible, the issues or problems that have yet to be solved.

\section{APPLICATIONS OF INDUSTRIAL YARNS}

Industrial twisted fibrous structures are mainly applied to load-carrying situations, and the applications can be classified into four different categories ${ }^{9}$ :

- Fastening operations: subjected to steady and/or cyclic loading; for instance, buoy moorings

- Transporting operations: subjected to a dynamic loading superimposed on a static tension-most marine applications such as towing vessels

- Lifting operations: subjected to a small number of dynamic cycles imposed on a steady loading, such as mounting climbing

- Impact operations: subjected to a falling object; impact energy must be absorbed without failure. Safety rope is an example.

In view of the nature of the applications, it is understandable that the mechanical durability and environmental attack resistance are the prime requirements for the performance of these materials.

\section{MATERIALS AND STRUCTURES}

Of all the twisted fibrous structures for industrial applications, ropes are probably the most complex in terms of structural compositions and application scenarios. Because ropes contain all forms of

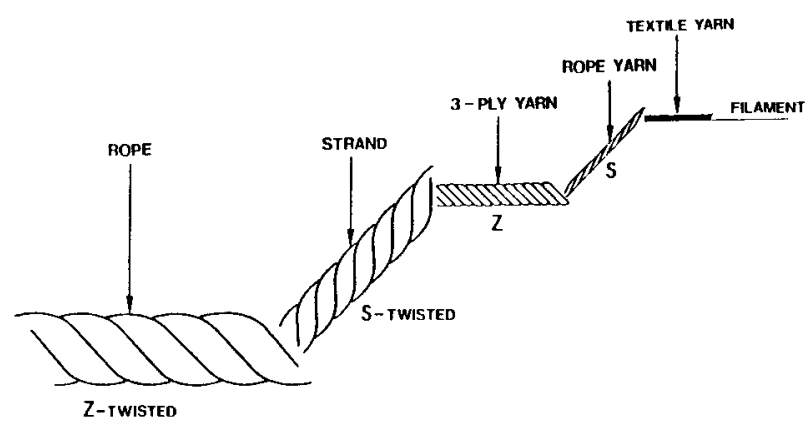

Figure 1 A rope structure. ${ }^{10}$

industrial twisted structures, in this article, they will be dealt with principally.

\section{Materials}

Marine ropes are composed of millions of individual fibers twisted and combined into many levels of substructures such as yarn, plied yarn, rope yarn, and strand (Fig. 1). The levels of structure are dependent on the complexity of the rope geometry. However, in principle, at least three levels can be identified, i.e., fiber, yarn, and strand.

In natural fibers, manila and cotton are common materials for rope, and in synthetic materials, nylon, polyester, polypropylene, and recently, Aramid (Kevlar $\left.{ }^{\circledR}\right)$ fibers are all good candidates as rope-making fibers. ${ }^{11}$ Table I provides more detailed information about the specifications of ropes made of different fiber types.

\section{Single Yarns and Helix Model}

As mentioned above, the fibers in a rope are twisted to various degrees to maintain the structural integrity, among other things. For filament yarns, the fiber arrangement in the structure is closer to the so-called idealized helical structures according to the description of Hearle et $\mathrm{al}^{5}$ : "The yarn is assumed to be circular in cross section, and composed of a series of concentric cylinders of differing radii. Each fiber follows a uniform helical path around the concentric cylinders, so that its distance from the yarn axis remains constant. A fiber at the center will follow the straight line of the yarn axis; but, going out from the center, the helix angle gradually increases, since the number of turns of twist per unit length remains constant in all the layers. The density of packing of fibers in the yarn remains constant throughout the model; and the structure is assumed to be made of 
Table I Comparison of the Performance of Some Ropes with a Strength of $500 \mathbf{k N}^{12}$

\begin{tabular}{llccr}
\hline \multicolumn{1}{c}{ Fiber } & Rope Type & $\begin{array}{c}\text { Weight } \\
(\mathrm{kg} / 100 \mathrm{~m})\end{array}$ & $\begin{array}{c}\text { Stiffness } \\
(\mathrm{kN} / 1 \% \text { ext })\end{array}$ & $\begin{array}{c}\text { Price } \\
(£ / 100 \mathrm{~m})\end{array}$ \\
\hline Manila & Three-strand & 500 & 40 & 600 \\
Steel & Galvanized & 300 & 360 & 700 \\
& Stainless & 300 & 360 & 2500 \\
Polyester & Double braid & 220 & 30 & 1500 \\
Polypropylene & 8-Strand & 200 & 20 & 500 \\
Nylon & Double braid & 150 & 15 & 1200 \\
Polyester & Parallel braid & 150 & 40 & 1000 \\
Carbon & Pultrusion & 70 & 600 & 10,000 \\
Aramid & Parallel yarn & 40 & 80 & 1600 \\
HMPE & Stranded & 40 & 80 & 1600 \\
\hline
\end{tabular}

a very large number of filaments, so that various complicating effects, which are due to the special ways of packing a limited number of fibers together, do not arise."

\section{Plied Yarns}

A plied yarn is formed by twisting together two or more single yarns, which is similar in many geometric aspects to the twisting of two or more filaments to form a single yarn. Instead of the fiber following the helical path, it is now the single individual yarn axis that lies along the helix.

\section{Twisted Rope Structure}

Ropes are formed by several strands each consisting of several plied yarns. For a twisted rope, the twisting direction in different structural levels must alternate between $Z$ and $S$ twisting to achieve a balanced structure.

\section{Braided Rope Structure}

Besides twisted ropes, another method of making rope is by using plaiting or braiding. This is a modified form of weaving yarns into a tubular form. The structure of braided rope is more stable than the twisted one; therefore, thicker and stronger ropes can be made this way. Also, by braiding the structure into a balanced structure, it "possesses the advantage that no torque is induced in rope stretched by virtue of the axially symmetric structure of the rope."13

\section{Fiber Migration}

The requirement of filament continuity and the practicality of equivalent filament feed rates into the twisting zone necessitate the interchange of filaments between coaxial helical rings, so that there will be a periodical change of radial location for each filament, which is termed migration.

As a result of both twisting and fiber migration, the path of a fiber orientation in a rope becomes rather complex; the geometry of double-braided rope was considered representable with a sinusoidal undulation superposed on a circular helix. ${ }^{14}$

\section{TENSILE BEHAVIOR OF THE STRUCTURES}

Twisted fiber structures have a unique strengthgenerating mechanism; the force that is breaking the structure is at the same time strengthening it. So the prediction of yarn tensile behavior is a complicated issue, and many factors and effects have to be considered.

\section{General Treatments and Models}

There have been several general treatments on yarn mechanics. Starting from a simple analysis by Gegauff, ${ }^{15}$ to a more complex model by Platt, ${ }^{16}$ Hearle ${ }^{17}$ Treloar, ${ }^{18}$ Kilby, ${ }^{19}$ and to the Wilson's model of a filament cord ${ }^{20}$ as well as Costello's ${ }^{21}$ analysis on wire ropes. Recently, Pan has published a series of articles on the general theoretical treatment of twisted fibrous structures. ${ }^{22-27}$

The modeling of a rope structure is more complex, and there have been some attempts only 
recently. ${ }^{28,29,13,14}$ Limited by the complexity of rope structures and their discrete components, the classical continuum mechanics approach is not readily applicable, and the structural modeling method is adopted instead.

When modeling a rope structure, two constraints have to be satisfied: 1 . the compatibility condition, where the total rope stretch equals the summation of stretch in different elements over a helix period; and 2 . the equilibrium condition, where the resultant axial force acting on any rope cross-section is constant along the rope axis.

For a given rope structure in which the strand's geometrical configurations are initially prescripted, the general approach of modeling follows the steps below ${ }^{13}$ :

1. The strand tensile properties and geometrical parameters are input factors

2. For each given axial rope load, a new rope geometry is determined

3. Computing the local length change at different locations along a strand yields a strain distribution in the rope strand

4. Strand load is then determined through its load-strain relationship

5. By converting the individual strand load into rope axial load and summing up the contributions from different strands, a rope load-strain curve is generated.

\section{Fiber Tension and Lateral Compression in a Yarn}

To understand and predict the strength of filament yarns accurately, the effect of transverse pressure on the individual fibers caused by tensioning of the yarn is considered.

Although there have been several versions of the expressions for yarn tension and lateral compression, Pan's ${ }^{23}$ results probably are the most concise. If we ignore the effect of fiber migration, then the fiber tension in a staple yarn can be written as

$$
\sigma=E_{f} \epsilon_{f}\left[1-\frac{\cosh \left(n x / r_{f}\right)}{\cosh (n s)}\right]
$$

where $E_{f}, \epsilon_{f}$, and $r_{f}$ are the fiber modulus, strain, and radius; $x$ is the distance from either fiber end to the center; $s=l_{f} / 2 r_{f}$ is called the fiber aspect ratio where $l_{f}$ represents the fiber length; and

$$
n=\sqrt{\frac{G_{T L}}{E_{f}} \frac{2}{\ln 2}}
$$

is an indicator of the gripping effect of the yarn structure on each individual fiber. It was therefore named the yarn cohesion factor and is related, as shown in the equation, to the ratio of yarn longitudinal shear modulus $G_{T L}$ and the fiber tensile modulus $E_{f}$, as well as the fiber arrangement within the yarn reflected by the analysis in Pan. ${ }^{23}$

The lateral compression on the fiber is

$$
g=\frac{n}{2 \mu} E_{f} \epsilon_{f} \tanh (n s)
$$

where $\mu$ is the interfiber frictional coefficient.

For a continuous filament yarn or a staple yarn in which twist level is high enough so that $n s \gg 1$, there $\operatorname{are} \cosh (n s) \rightarrow \infty$ and $\tanh (n s) \rightarrow 1$. We then have

$$
\sigma=E_{f} \epsilon_{f}
$$

and

$$
g=\frac{n}{2 \mu} E_{f} \epsilon_{f}
$$

The importance of lateral pressure on load transfer from fiber to fiber in a twisted fibrous structure was recognized as far back as Galileo, ${ }^{30}$ and several researchers in yarn mechanics have incorporated the effect of the transverse force into their analytic models. For example, Gurney ${ }^{31}$ developed a relationship between yarn strength, twist level, lateral contraction ratio, and fiber stress-strain properties. Sullivan ${ }^{32}$ determined the strength of staple fiber yarns by analyzing the lateral pressure distribution across the yarn and its contribution to axial stress. Machida ${ }^{33}$ analyzed the lateral pressure in blended filament yarns under tension as a means of predicting the recovery fiber length during yarn rupture. Grosberg $^{34}$ calculated the lateral pressure distribution in a low-twist sliver under extension so as to predict the sliver strength. Dogu ${ }^{35}$ derived the distribution of transverse pressure in a twisted yarn including fiber migration and fiber packing density variation. Hearle, ${ }^{5}$ as well as Kilby ${ }^{19}$ and White et al. ${ }^{36}$ provided the comprehensive analysis of filament yarn strength taking into account transverse forces, leading to a more complete understanding of the relationship between properties of filament and yarn. 


\section{Fiber Bending and Torque in a Yarn}

For textile structures, bending is involved not only in their forming processes, but actual use conditions may include various modes of bending motion. For instance, when a rope is stretched, the yarn deformation not only includes tensile, but also bending and torsional deformations because of the helical orientation of the fibers. Thus, bending effects have been an active research area for many years and good progress has been achieved in several studies; for instance, early work by Backer $^{37}$ in yarn bending geometry, Platt $^{16}$ in calculating yarn bending rigidity, and Zorowski and $\mathrm{Chen}^{38}$ in frictional constraints. Kim and coworkers ${ }^{39}$ investigated the bending properties of monofilaments at large bending deformation (beyond yielding) and considered the filaments to be elastic-plastic. Wu et al. ${ }^{13}$ studied the rope behavior as a bending field superimposed on a tension strain field. Marine ropes are usually subjected to bending by virtue of geometric constraints such as eye splice terminations or looping on a pin, or running through pulleys and blocks.

Twisting a yarn is very different from twisting a solid shaft. "The linear elastic analysis of twisting a rod to a small strain reveals that the primary mode of deformation of the rod material is that of pure shear. However, when a yarn is twisted, there is no significant resistance to the fibers that lie parallel in the yarn simply sliding past each other in the direction of the fibers. In other words, there is virtually no resistance to immediate and catastrophic shear failure. Resistance to twisting is very low in any case and it only builds up significantly at large strains."

\section{Fiber Compression Behavior in a Yarn}

Industrial cord is designed as a structural element dealing with relatively large axial loads in tensile in comparison to bending and torsional loads. However, sometimes a lateral compressive load may be added upon the cord. When a cord is subjected to such compression, the individual filaments lose contact with each other. This results in a considerable reduction in the axial stiffness of the cord. ${ }^{40}$

\section{Yarn Constitutive Equations and Elastic Constants}

One of the fundamental tasks in studying the mechanical behavior of a medium is to derive its stress-strain or constitutive relationships. In the case of the ideal yarn model defined above, the fibers can be assumed to be arranged in the transverse plane in such a manner that there is no preferential packing geometry in the plane, thus making the plane mechanically isotropic and allowing the yarn structure to be treated as a transversely isotropic material. Consequently, its stress-strain relationships can be expressed by the following equation ${ }^{8}$ :

$$
\left[\begin{array}{c}
\epsilon_{11} \\
\epsilon_{22} \\
\epsilon_{33} \\
\gamma_{12} \\
\gamma_{23} \\
\gamma_{31}
\end{array}\right]=\left[\begin{array}{cccccc}
\frac{1}{E_{T}} & -\frac{\nu_{T T}}{E_{T}} & -\frac{\nu_{L T}}{E_{L}} & 0 & 0 & 0 \\
-\frac{\nu_{T T}}{E_{T}} & \frac{1}{E_{T}} & -\frac{\nu_{L T}}{E_{L}} & 0 & 0 & 0 \\
-\frac{\nu_{T L}}{E_{T}} & -\frac{\nu_{T L}}{E_{T}} & \frac{1}{E_{L}} & 0 & 0 & 0 \\
0 & 0 & 0 & \frac{1}{G_{T T}} & 0 & 0 \\
0 & 0 & 0 & 0 & \frac{1}{G_{T L}} & 0 \\
0 & 0 & 0 & 0 & 0 & \frac{1}{G_{T L}}
\end{array}\right]\left[\begin{array}{l}
\sigma_{11} \\
\sigma_{22} \\
\sigma_{33} \\
\tau_{12} \\
\tau_{23} \\
\tau_{31}
\end{array}\right]
$$

where 1,2 , and 3 refer to the $X_{1}, X_{2}, X_{3}$ directions in the Cartesian coordinate system and $E_{L}$ is the longitudinal modulus governing uniaxial loading in the longitudinal $\left(X_{3}\right)$ direction, $\nu_{L T}$ is the associated Poisson's ratio governing induced transverse strains, $E_{T}$ is the transverse modulus governing uniaxial loading in the transverse $\left(X_{1}\right.$ or $X_{2}$ ) direction, $\nu_{T L}$ is the associated Poisson's ratio governing induced longitudinal strains and $\nu_{T T}$ is the associated Poisson's ratio governing resultant strains in the remaining orthogonal transverse ( $X_{1}$ or $X_{2}$ ) direction, $G_{T L}$ is the longitudinal shear modulus governing shear in the longitudinal direction, and $G_{T T}$ is the transverse shear modulus governing shear in the transverse plane.

A comprehensive description of the mechanical behavior of a staple yarn relies on the determination of these material constants.

There have been several attempts in deriving the constants, and the following is the result from Pan. ${ }^{23}$ For an ideal yarn model of continuous filaments, we have the modulus in longitudinal direction

$$
E_{L}=\frac{3 V_{f} E_{f}}{4} \Theta_{1}(q)=\frac{3 V_{f} E_{f}}{4} \frac{(1+\cos q)^{2}}{1+\cos q+\cos ^{2} q}
$$

where $E_{f}$ and $V_{f}$ are the fiber tensile modulus and fiber volume fraction in the yarn and $q$ is the helix angle at yarn surface. The transverse modulus is 


$$
\begin{aligned}
E_{T} & =\frac{8 V_{f} E_{f}}{\pi^{2}} \Theta_{2}(q) \\
= & \frac{8 V_{f} E_{f}}{\pi^{2}} \frac{(q / 2-1 / 4 \sin 2 q)^{2}}{\left(2 / 3-\cos q+1 / 3 \cos ^{3} q\right)(1-\cos q)}
\end{aligned}
$$

and the Poisson's ratios

$$
\begin{gathered}
\nu_{L T}=\frac{\sin ^{5} q}{2\left(1-\cos ^{3} q\right)(q / 2-1 / 4 \sin 2 q)} \\
\nu_{T L}=\frac{16 \sin q(q / 2-1 / 4 \sin 2 q)}{3 \pi^{2}\left(2 / 3-\cos q+1 / 3 \cos ^{3} q\right)} \\
\nu_{T T}=\frac{2}{\pi}
\end{gathered}
$$

as well as the shear modulus

$$
\begin{aligned}
G_{T T} & =\frac{4 V_{f} E_{f}}{\pi(2+\pi)} \Theta_{3}(q) \\
& =\frac{4 V_{f} E_{f}}{\pi(2+\pi)} \frac{(q / 2-1 / 4 \sin 2 q)^{2}}{\left(2 / 3-\cos q+1 / 3 \cos ^{3} q\right)(1-\cos q)}
\end{aligned}
$$

The shear modulus $G_{T L}$ is

$$
\begin{aligned}
& G_{T L}= E_{f} V_{f} \Theta_{4}(q) \\
&=E_{f} V_{f} \frac{1}{\frac{\pi(1-\cos q) \sin ^{3} q}{6(q / 2-1 / 4 \sin 2 q)^{2}}} \\
& \quad+\frac{8 \sin ^{3} q}{3 \pi(1-\cos q)(1+\cos q)^{2}} \\
& \quad+\frac{\pi\left(4-3 \cos q-\cos ^{3} q\right)}{6(q / 2-1 / 4 \sin 2 q)(1+\cos q)}
\end{aligned}
$$

That is, all the material moduli are the functions of fiber modulus, fiber volume fraction, and yarn twist as reflected by the surface helix angle $q$, and the system Poisson's ratios are only determined by the surface helix angle $q$. For staple fiber with limited length and hence fiber slippage, the results have to be modified. ${ }^{23,24}$ Also, the results above only consider the effect of fiber extension, and bending effect is incorporated into the results in Pan. ${ }^{25} \mathrm{~A}$ more complete treatment of the problem by Thwaites can be found in Ref. 6 where torsion effects are also included.

\section{BLENDED STRUCTURES}

Fiber blending has been a long-time common practice in textile processing. By mixing fibers of different types to form textile yarns, many advantages are achieved such as property compensation or reinforcement between fibers, cost reduction without significant sacrifice of yarn performance by partially replacing expensive fibers with less expensive ones, and cross-dyeing effect caused by the different dye affinity of multiple fiber types. Owing to the importance of blended yarn structures, it is desirable to understand and specify their mechanical behavior to realize the potential of the blending process.

The investigation of the mechanics of blended yarns has been the topic of many studies, ${ }^{26,41-47}$ and most of these studies have focused on the prediction of the tensile strength of the yarn as the most important yarn property with practical significance. Considering the complexity of the mechanics of staple yarns with even a single fiber type, the existence of multiple fiber types in a twisted structure adds a formidable dimension to the theoretical analysis.

\section{Intimate Blending and Structural Blending}

There are several ways to blend fibers into ropes, i.e., intimate and structural blendings. Intimate blending is the case in which different fiber types are mixed uniformly in the system, whereas the different fiber types concentrate at different locations in the system in the case of structural blending. "It is common, for example, for a jacket to be made of polyester around a high-modulus fiber core. There can also be intimate blends. In ropes that are subject to high loading, a polyester/ polypropylene blend can minimize the effects of heating. At a temperature when the polypropylene melts, absorbs heat and prevent further temperature rise, the polyester remains effective."12

\section{Problems Associated with Fiber Blending}

There are several aspects that make hybrid structures much more difficult to analyze. In hybrid yarns, in which two different types of fibers are blended together to form a system, the differences in their contributions toward the overall behavior of the structure, because of the diverse mechanical properties of the constituent fibers, have to be 
considered. Secondly, the interaction between the two constituents will alter the nature of yarn behavior, especially during fracture. Inclusion of this interaction into analysis has proven to be very challenging.

\section{The Hybrid Effects}

One phenomenon associated with the interaction in a blended structure that greatly complicates analysis is the so-called "hybrid effect," which is defined in Marom et al. ${ }^{48}$ as the deviation of behavior of a hybrid structure from the Rule of Mixtures. A positive hybrid effect means that the property is above the prediction given by the Rule of Mixtures, whereas a negative hybrid effect means the property is below the prediction. There is a second definition for the hybrid effect as the difference between the performance of a fiber in a hybrid structure and in a nonhybrid structure ${ }^{49,50}$ Again the hybrid effect can be positive or negative depending on whether the property in the hybrid system is greater or smaller than that in the nonhybrid system.

In previous articles by Pan and Postle, ${ }^{51,52}$ three physical mechanisms have been identified and demonstrated to be responsible for causing the hybrid effects in a hybrid structure. The first (Mechanism I) is the protection or enhancement afforded by the system to the fibers through the so-called fragmentation process during structure extension. This enhancement effect is largely dependent on the along-fiber property variation (variation along a fiber length) and on the fiberfiber gripping. Because of this enhancement, the (in situ) behavior of the fibers in a structure will be different from the original (ex situ) fiber behavior determined before the fibers are embedded into the system. The second mechanism (Mechanism II) is related to the between-fiber property variation, i.e., variation of breaking properties between fibers of the same type. Because of this variation, fibers of the same type in the structure will break gradually according to the statistical distribution of their breaking strain, eventually reducing the values of strength and breaking elongation of the structure because of the fiberfiber interaction of the same type. Because the along-fiber property variation and the betweenfiber property variation exist in both hybrid and nonhybrid systems, Mechanisms I and II are effective in a yarn of single-fiber type as well.

The third (Mechanism III) is the cross coupling effects between the different fiber types in the structure; this mechanism was found ${ }^{52}$ in general to enhance the fiber in situ properties of the lower-breaking-strain fiber type but depresses those of the higher-breaking-strain fiber type, leading to a positive hybrid effect associated with the first peak and a negative hybrid effect associated with the second peak on the stress-strain curve of a blended structure, when compared with nonblended structures.

\section{YARN FRACTURE AND TENSILE STRENGTH}

Strengths of yarns are undoubtedly the most important yarn properties, because yarns have to be durable enough to sustain the various actions during the subsequent process of applications. For this reason, research work to understand the strength-generating mechanism, and to predict yarn strength has been one of the most productive areas in textile literature. ${ }^{5,16,27,32}$

It is well known that, because of the variations in fiber strength, the breaking stress of a parallel fiber bundle deviates from that of its constituent fibers. However, prediction of strength of a twisted fiber structure is also different from that of a parallel fiber bundle because, in the latter case, the effect of fiber interaction is negligible, and also as fibers are all parallel to the axis of the assembly (the loading direction) in the parallel bundle, the fiber obliquity effect is nonexistent. Moreover, strength prediction of a fibrous structure is unlike its modulus; the strength of a material is not a volume-average quantity but rather an extremum quantity, dictated by the weakest cross-section of the structure. This so-called weakest link theorem was first elucidated by Peirce $^{53}$ in 1926 and has since been thoroughly discussed by numerous authors.

Daniels $^{54}$ demonstrated that, if the fiberstrength distribution is of Weibull ${ }^{55}$ form, the asymptotic strength distribution of a parallel fiber bundle when the bundle size $N$ is large enough is of normal type. This conclusion has been accepted by the latter studies. Harlow and Phoenix ${ }^{56}$ proposed the concept of the chain-of-bundles model of the strength of fibrous structure to tackle the issue of statistical nature of strength of individual filament, the size (length) effect on filament strength as well as the load-sharing mechanism during structure breakage. Phoenix ${ }^{57}$ also extended their method to the analysis of twisted fiber bundles by incorporating the fiber helical paths into his model. However, exclusion of the 
effects of fiber interaction such as interfiber friction and lateral constraint in his model brings serious limits to his theory in terms of the applicability and the accuracy of prediction.

\section{Interfiber Friction and Interactions}

Friction is the chief mechanism by which any textile structure is able to assemble; it is therefore fundamental in our study of yarn behavior to look into the detailed frictional behavior between fibers in a textile structure. There have been many studies on friction in textiles. ${ }^{58-60}$

To better understand and more accurately predict the strength of twisted fiber structures, besides the properties of the constituent fibers, the interactions between fibers as well as between the fiber and yarn structure have to be considered, because these interactions will significantly alter the in situ fiber properties. Research on the prediction of yarn strength hence involves the investigation of the flaw distribution along the fiber length and its effect on yarn strength, the fiber in situ mechanical properties, and the stress transfer from the broken fiber into still-surviving fibers during yarn extension. Some of these issues are also of interest for researchers in fiber composite materials, and some are fundamental to materials science in general.

\section{Fragmentation and the Critical Length}

Besides the weakest link mechanism, there is another mechanism known as the fragmentation process which relates to, but acts differently from, the weakest link mechanism. It was observed during the fracture process of both composites ${ }^{61}$ and yarns ${ }^{45}$ that the constituent fibers break repeatedly along the length with increasing strain of the structure before overall material failure. This phenomenon indicates a fact that, contrary to common assumption, a broken fiber can again build up tension, carry load, break into even shorter segments, and contribute toward overall system strength. Also, the fiber breaks will not stop, as long as the whole structure does not collapse, until the length of the breaking segments reaches a minimum value at which its load can no longer build up to its segment breaking strength. This length is well known as the critical length $l_{c}$. If $\sigma_{b}$ is the tension that causes the fiber segment to break, it follows that the minimum length on which a broken segment can no longer build up its tension again from the broken position or the minimum length into which a fiber can be broken is $^{33}$ :

$$
l_{c}=\frac{\sigma_{b}}{\pi r \mu g}
$$

where $\mu$ is the frictional coefficient, $r$ is the fiber radius, and $g$ is the local lateral pressure.

However, because of the length-strength dependency implied by the weakest link theory, the strengths of these fiber segments will become higher with decreasing length. It was reported by Pan et al. ${ }^{22}$ that even excluding the fiber obliquity effect, which will lower the contribution of individual fibers toward the yarn strength, the experimentally determined yarn breaking load is still greater than the prediction based on the predetermined breaking force of all its fibers. A new mechanism was proposed to account for this discrepancy. Given the fact that the shorter fiber will show a higher breaking strength, it was suggested that, because of the fragmentation process, the twist-induced lateral pressure substantially raises the overall strength of a filament yarn by increasing the apparent strengths of the segments of each individual fibers. This also suggests the assistance of a structure to its constituent components during loading.

Yet, there is a difference in the fragmentation process between fiber composites and yarns. For a fiber composite, because the bond adherence between fibers and the matrix remains largely unchanged as tension on the composite is increased, the critical fiber length that determines the final length of the fiber fragments can be treated as constant. However, in the case of twisted yarns, because the lateral pressure in the yarn that provides lateral constraint on fibers is dependent on the external tensile loading, the length of the fiber fragments hence changes (decreases) along with the increasing lateral pressure during yarn extension up to the ultimate yarn failure. This mechanism will vary for different yarn structures and will also be strongly influenced by the geometric, mechanical, and surface properties of fibers (including their variations). ${ }^{22}$ All of this considerably complicates the analysis of yarn strength.

It should be pointed out that the significance of the fiber fragmentation effect is dependent on the differences between the breaking strains of the structural components. Although this effect is more noticeable in fibrous structures in which the breaking strains of the structure components (fi- 
ber and matrix material in composites or distinct fiber types in blended yarns) are remarkably different, it should also play a role in other fibrous structures as long as there is a dispersion in fiber breaking strain or strength.

\section{Load Sharing Between Fibers}

Another important aspect of interfiber interactions is the so-called load sharing process. During yarn failure, the fibers in the yarn will not break simultaneously. Instead, there exists a span over yarn strain during which fibers fail individually because of the variations caused by many factors. The ones still surviving have to share the load previously carried by the just-broken ones. This load-sharing mechanism varies based on yarn structures, fiber types, and twist levels. Harlow and Phoenix ${ }^{56,57}$ have dealt with the this problem extensively, for both yarns and fiber-reinforced composites. It is understandable that the loadsharing process in a composite is more local because of the relatively strong fiber-matrix chemical bonding.

\section{In Situ and Ex Situ Fiber Properties}

Because of the effects of interfiber interactions, it is often meaningless to deal with the mechanical properties of the constituent fibers isolated from a specific structure because these (ex situ) properties will alter once the fibers are assembled into the structure. This change of properties (the in situ properties) may be caused by two factors; first, the fibers incorporated into a structure are under a complex loading situation because of interactions; second, the fragmentation process reduces the fiber length, and consequently increases the fiber strength. It may be desirable to designate new conditions (close to the real situation of the structure) under which the properties of the fibers will be determined.

\section{STRAIN RATE, VISCOELASTICITY, DYNAMIC, AND FATIGUE EFFECTS}

\section{Viscoelastic and Strain-Rate Effect}

There are a limited number of studies in this area in textile literature. Huang studied the viscoelastic tensile behavior of a two-ply filament yarn ${ }^{62}$ and multi-ply yarn. ${ }^{63}$ Asvadi and Postle ${ }^{64}$ have used linear viscoelastic theory dealing with the large strain shear problem. There have been more extensive investigations on time-dependent, or viscoelastic behavior of cord or rope structures in the mechanical engineering field by Conway and Costello. ${ }^{65-67}$ They proposed a new method in which the axial viscoelastic response of simple strand may be predicted given the stress relaxation of the filament's construction material without considering the effects of friction and material compliance.

\section{Dynamic Loading}

El-shiekh ${ }^{68}$ published a work dealing with the dynamic modulus of viscose-polyester blends. Recently, Vangheluwe and Goswami ${ }^{69}$ published a note on the strain rate in dynamic tensile testing. Wu et al. ${ }^{13}$ observed that, at dynamic tensile loading situation, a rope structure becomes more compact, and "its stiffness increases while it retains the same failure strength, but it experiences a much lower strain to failure. The magnitude of change in dynamic behavior rises sharply in the beginning of load cycling, but soon reaches a stable value."

\section{Fatigue and Environmental Attack}

Anandjiwala et al. ${ }^{70,71}$ have done considerable work dealing with the fatigue behavior of staple fiber yarns. From the pathological studies of used and tensile-cycled rope, it was concluded by Seo et al. ${ }^{14,72}$ that tensile creep and wear (internal and external) processes are two major rope deterioration processes under environmental attack. From friction and wear tests of monofilaments and rope yarns, it was determined that the wear life of yarn is dominated by the total number of friction cycles.

\section{SOME SPECIFIC PROBLEMS AND ISSUES}

Six specific topical areas are identified in this part for further discussion, namely, the nature of the fibrous structures; the fiber packing problem; the stress transfer problem; the fiber structure fracture and failure problem, and the hybrid structure problem; and the size or scale problem. These problems are applicable, but not limited, to twisted fibrous structures. It is our opinion that the progress in all of these areas has reached a point that they should each be treated as an individual topic for more in-depth research. They 
represent, in fact, some of the fundamental issues in mechanics of fibrous structures.

\section{Nature of the Fibrous Structures}

A fibrous structure is chiefly a mixture of fiber and air so that the overall system behavior is determined by the properties of each constituent and any possible coupling effects between them.

\section{Fibrous Structures as Discrete Media}

Fibrous structures are not classical continuum, but rather are discrete because of the existence of the macro-pores. Micromechanics is often used to study the mechanical behavior of discrete media from microstructural considerations and is based on the properties of its constituents. However, the inherent random nature of the physical and geometrical features of discrete media is fundamentally different from the macroscopic level of the assumed continuum, when the method of combined microanalysis and continuum theory is used. Therefore, the connections between the formulations from the microstructural analysis and the macroscopic performance have to be established as the premises for the discrete media study. Axelrad ${ }^{73,74}$ has proposed that, in the formulation of the mechanics of a discrete medium, three measuring scales should be used to define such a system. The smallest scale is called a "microelement" of the structure. It is a typical representative element of the microstructure of the system on which all of the continuum concepts are applicable, as it is a continuum by definition. Then, an intermediate scale named "mesodomain" containing a statistical ensemble of the microelements follows. The physical and geometrical parameters of the mesodomain are independent of the positions, and have to be derived statistically based on the parameters of its constituent microelements. In fact, the mesodomain is defined as a portion of, or as the representative of, the whole system on which the continuum approach is once again valid, provided that only the effects over distances appreciably greater than the distance between the microelements are concerned. ${ }^{8}$ Finally, a finite number of nonintersecting mesodomains form the macroscopic material body. These three divisions clearly illustrate the relationships between the different structural (from microscopic to macroscopic) levels, and thus, actually provide the natural sequence of the micromechanical analysis.
The concepts of these three divisions have been applied, consciously or subconsciously, by previous researchers in dealing with fibrous systems. ${ }^{75-78}$

\section{The Genuine Stress and Strain for Fibrous Structures}

Furthermore, because of the porous nature of the fibrous materials, the conventional mechanical definitions such as stress and strain may not be entirely appropriate to be applied to the materials. For instance, when a pressure is exerted onto a textile surface, the effective surface area that provides resistance to the load is not the total area on which the pressure is on, because the area consists of fiber and voids, and the latter should be eliminated in normal situations. Also, when this material is deformed, the total deformation again includes those of both fiber and voids. In other words, the genuine stress and strain for fibrous structures are the ones that preclude the effect of the voids, or at least deal with the voids and fiber separately and differently. This is not a simple matter of changing the definitions, and it might have profound implications on our understanding of the behavior of fibrous systems.

\section{Fiber Packing}

Fibers are not isotropic materials, having distinct properties between the axial and transverse directions. They are best performed when dealing with axial tension, but behave not as well in all other loading situations such as compression and shearing. Also, various loads have to be transfered through the fiber-fiber contact points. Therefore, the format in which the fibers are arranged in a structure becomes a critical issue; it will determine virtually all the properties of the system.

\section{Fiber Packing Problem}

The problem of fiber packing was initially studied by van $\mathrm{Wyk}^{79}$ in his analysis of the compressibility of wool by looking into the geometrical characteristics of a fiber mass formed by fiber packing. Komori and Makishima have examined the details of such geometrical features in a fiber assembly, including the mean fiber contact density and the mean fiber length between two fiber contacts, ${ }^{80}$ and the fiber and pore distribution in the fiber assembly. ${ }^{81,82}$ Lee et al. ${ }^{83}$ have published an article to review the research progresses in this 
field. More recently, $\operatorname{Pan}^{84}$ has considered the steric hindrance effect, i.e., the interference of existing fiber contacts on the successive new contact to be made. Komori and Itoh ${ }^{85}$ subsequently published a study to treat the same problem but to allow the system volume to change so that there become two competing factors affecting fiber contact. On the one hand, an existing fiber contact reduces the effective contact length of a fiber and hence diminishes the chance for new contacts. On the other hand, the existing fiber contact point will also abate the free volume of the fiber mass, and consequently increase the chance for successive fibers to make new contacts. Some of the research results in this area have been applied to study the compressional ${ }^{75,83}$ and shear $^{86}$ behavior of general fiber assemblies, as well as the prediction of nonwoven products, ${ }^{77}$ leading to considerable progress in those areas.

Nevertheless, research on this problem is still very elementary. To understand the behavior of fibrous structures, one must examine the microstructure or the discrete nature of the structure. However, a thorough study of a structure formed by individual fibers is an extremely challenging problem. It is worth mentioning that the problem of the microgeometry in a fiber assembly can be categorized into a branch of complex problems in mathematics called packing problems. Consider, for example, the sphere-packing problem, also known as the Kepler problem, which has been an active area of research for mathematicians since it was first posed some 300 years ago, and remains unsolved. ${ }^{87}$ Yet, it seems that the sphere packing would be the simplest packing case, for one only needs to consider one characteristic size, i.e., the diameter of perfect spheres, and ignore the deformation caused by packing. Therefore, it does not seem that the fiber-packing problem can be solved completely anytime soon.

\section{Fiber Orientation Effects}

Because fibers are not isotropic, the fiber orientation thus has a determining effect on the system properties. In most twisted fibrous materials, fibers are oriented in a certain range of directions, and the system therefore has different properties at different directions.

It is considered common sense for textile scientists that when some fibers of modulus $E_{f}$ are oriented in a direction different from the yarn axis by an angle $\theta$, if ignoring the Poisson's effect, then the contribution of these fibers toward yarn modulus $E_{y}$ is the well-known law

$$
E_{y}=E_{f} \cos ^{2} \theta
$$

This can be more rigorously proven by using tensor transformation. However, it should be noted that not all fibers in a yarn are oriented in the same direction, but rather are distributed over a range, and the final result should be from an integration over the whole range of the fiber orientation, and dependent on the fiber orientation function as shown previously. ${ }^{88}$ The simple $\cos ^{2} \theta$ is only an approximation at best.

\section{Stress Transfer Problem}

For a continuous filament yarn, external tension can be applied directly to each of the filaments. But this is not the case in a staple yarn; the tensile load can only be exerted at both ends of the yarn and the stress has to then be transmitted to each of the individual fibers through a so-called "shear lag" mechanism, proposed by Cox, ${ }^{89}$ via interfiber friction generated through twist as shown in equations derived by Pan. ${ }^{23}$ This stress transfer also occurs in filament yarn during the so-called fragmentation process. Because of this stress transfer process, several issues have to be considered to determine the yarn properties.

The staple fibers are assembled into a continuous strand by virtue of twist alone. The tension in the fiber is built up from zero at the fiber ends to the maximum somewhere along the fiber length, ideally at the center. The tension distribution along the fiber length is linear at the portion of fiber length where slippage takes place. But at the portion of fiber tightly gripped through interfiber friction, a hyperbolic tension distribution has been proposed by Pan, as in eq. 1. Other issues, including the distribution of the frictiongenerated shear stress within a yarn, the fiber length effects on yarn properties, and the critical twist level above which a self-locking mechanism is formed so that a staple yarn gains certain strength, were all analyzed previously. ${ }^{23,24}$

However, several complex problems have yet to be solved. First, in all of the existing analyses, a continuous fiber-to-fiber contact in a yarn is assumed. Yet, in a more realistic case, fibers are in discrete point contact. This will completely alter the distributions of both the tension and shear stress in individual fibers. Also, several competing factors are involved in the prediction of the optimal twist level at which a staple yarn acquires the maximum strength, including the twist 
level, the fiber volume fraction of the yarn, the statistical variations, and the fragmentation process of yarn fracture behavior as discussed in next section.

\section{Fracture and Failure Problem in a Fibrous Structure}

Because of the discrete nature and the unique means by which individual fibers are assembled into a fibrous structure, their fracture process and failure criterion are distinctly different from those established for conventional engineering materials treated mechanistically as continuum.

\section{The Coulomb Yield Criterion and the Fiber Pullout Force}

In research of granular materials such as soil, rocks, or any other porous media made of particles assembled together through mainly cohesion and internal friction, there has been a well-known Coulomb yield criterion ${ }^{90}$ in predicting the shear failure of the system, i.e., the shear strength of the system $\tau_{u}$ is determined by the frictional $\mu \sigma$ and the cohesional resistances $c$, respectively, i.e.,

$$
\tau_{u}=\mu \sigma+c
$$

where $\sigma$ is the normal force acting on the system.

However, Grosberg and Smith ${ }^{34,91}$ have shown that if one pulls a fiber from a twistless sliver, the withdrawal force $W F$ per unit length of fiber required is given by

$$
W F=\mu^{\prime} P+W F_{O}
$$

where $P$ is the external pressure applied to the fiber mass; $\mu^{\prime}$ is the equivalent frictional coefficient but with dimensions of length, and $W F_{O}$ is the value for $W F$ when $P=0$, that is, the cohesion force. This conclusion has been utilized by Carnaby and $\operatorname{Pan}^{75,86}$ to predict the compressional hysteresis and the shear modulus of fiber assemblies.

Considering that eqs. 16 and 17 were independently derived, the similarity between them is both striking and inspiring: it highlights the discrete nature of fibrous structures. Therefore, textile structures cannot be treated strictly as continuum if one wants to study the local response of the structures under external loading.

Besides the fiber pullout test on a twistless sliver done by Grosberg and Smith, ${ }^{34,91}$ fiber pull- out from a bonded structure (nonwoven) and yarn pullout from a woven fabric have been studied by Pan, ${ }^{92}$ and Pan and Yoon ${ }^{93}$ theoretically and, for the latter case, also experimentally. For both structures, the fiber-fiber or yarn-yarn interactions are proven to consist of two forms; one is of an adhesive (for nonwoven fabric) or cohesive (for woven fabric) nature and is independent of the pressure at the crossing point, and the other is frictional and is directly related to the normal pressure that can be generated from tensions applied to the system. Yet, the cohesive force for woven fabric has proved to be generally negligible.

\section{The Failure Criterion for Textiles}

Because normally fibrous structures can only sustain tensile load, the failure criterion for linear fibrous material like yarns is very simple. One can use either the tensile breaking strain or the tensile strength. However, for planer fibrous materials such as paper and nonwoven and woven fabrics, the failure determination is not as straightforward, simply because the tensile strength of these materials is dependent on the loading format.

Prediction of fabric strength under complex loading has its significance both theoretically and practically. Fabrics are not only highly anisotropic, but also dimensionally unstable, very susceptible to external loading and to its historical situation. The important fabric properties critical to structural applications include the tensile strengths, the in-plane shear strengths, and the normal compressive (in thickness direction) strength, as well as the in-plane compressive strength, better known as the buckling strength.

The Tsai-Wu failure criterion ${ }^{94}$ is utilized by Pan and Yoon ${ }^{95}$ to woven fabrics, assuming it is valid for woven fabrics at least at the first quadrant where failure stresses are all tensile. The unknown coefficients in the failure criterion are determined based on the experimental results. The fabric shear strength, which is an important property of fabrics in load-carrying applications but is not very convenient to obtain experimentally, is predicted based on the measured uniaxial tensile strengths of the fabric at the principal and the off-axial directions. The influences of the various directions of the off-axial tensile test on the predictions of the fabric shear strength are also studied. 


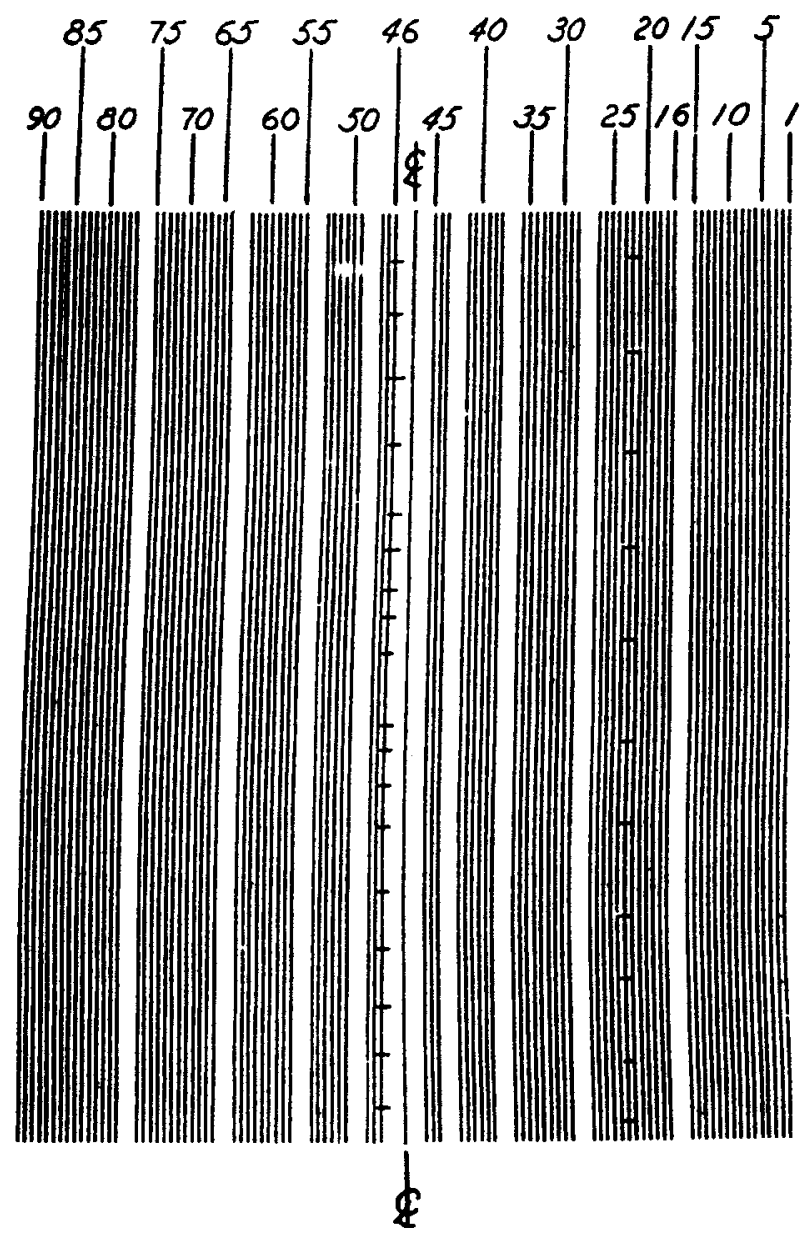

(a)

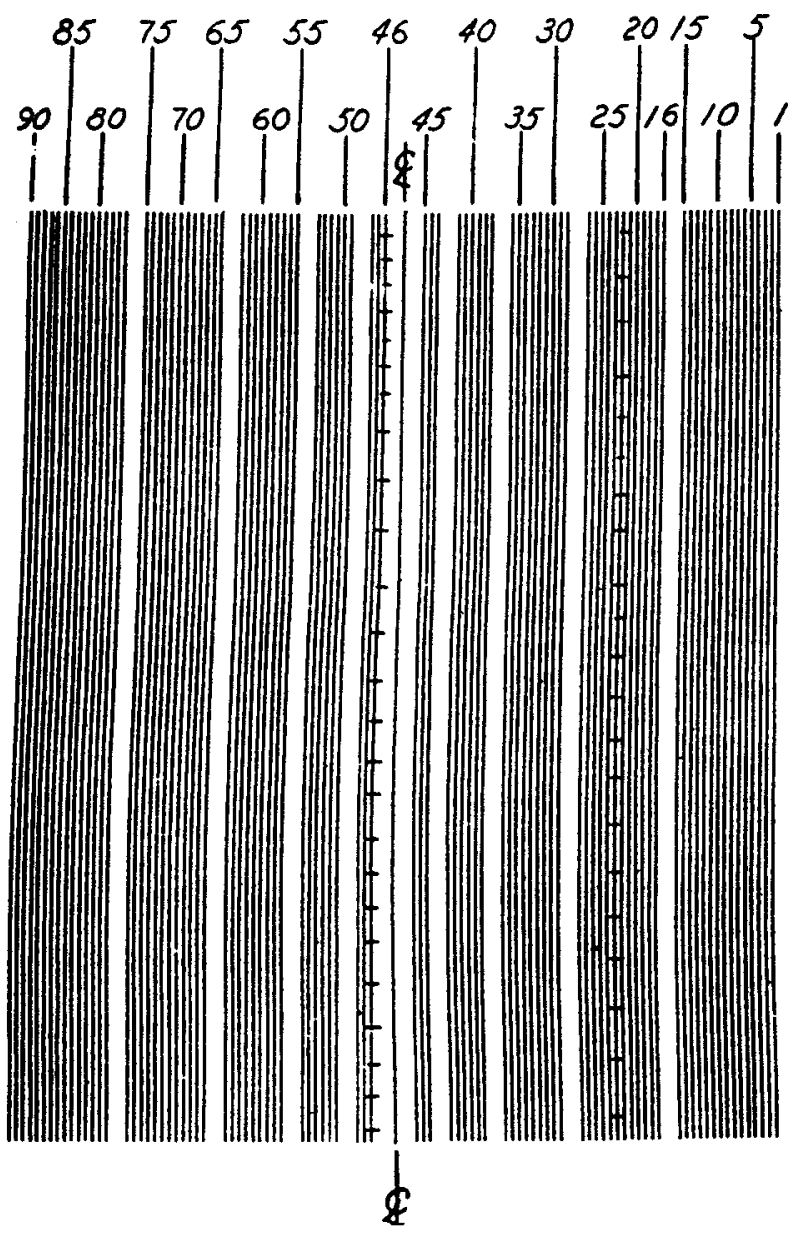

(b)

Figure 2 Experiments of a model with 2 cotton and 89 dacron components at TM $=2.19^{99}$ : (a) multiple breaks at $11 \%$ extension; (b) multiple breaks at $15 \%$ extension; (c) multiple breaks at $25 \%$ extension; and (d) yarn cross-section and its load-extension curve.

\section{Hybrid Structure Problem}

As mentioned above, in blended fibrous structures, the effects caused by the discrepancies in the properties of different fiber types as well as the mutual interactions between the fiber types have profound influence on the overall mechanical behavior of the system. Two cases can be identified, i.e., with or without the effects of statistical variations in fiber properties.

\section{The Mechanics of Blended Yarns Without the Statistical Variations}

$\operatorname{Pan}^{26}$ has performed a thorough study on the prediction of the elastic material constants such as the longitudinal and transverse tensile moduli, the shear moduli, and the Poisson's ratios of blended short-fiber yarns, based on the theory developed previously ${ }^{23}$ for yarns of single-fiber type by considering the structures as transversely isotropic, and combined with the techniques used in dealing with hybrid composite materials. The mean tensile strengths of the blended staple yarns are also predicted, with the exclusion of the statistical and the hybrid effects to begin with. The so-called minimum blend ratio, below which the mean yarn strength will definitely not follow the rule-of-mixtures, and the critical blend ratio, below which the mean yarn strength will be weakened rather than strengthened because of the addition of the reinforcing fibers, are calculated and the variables involved are discussed. Finally, the effects of the breaking strains be- 

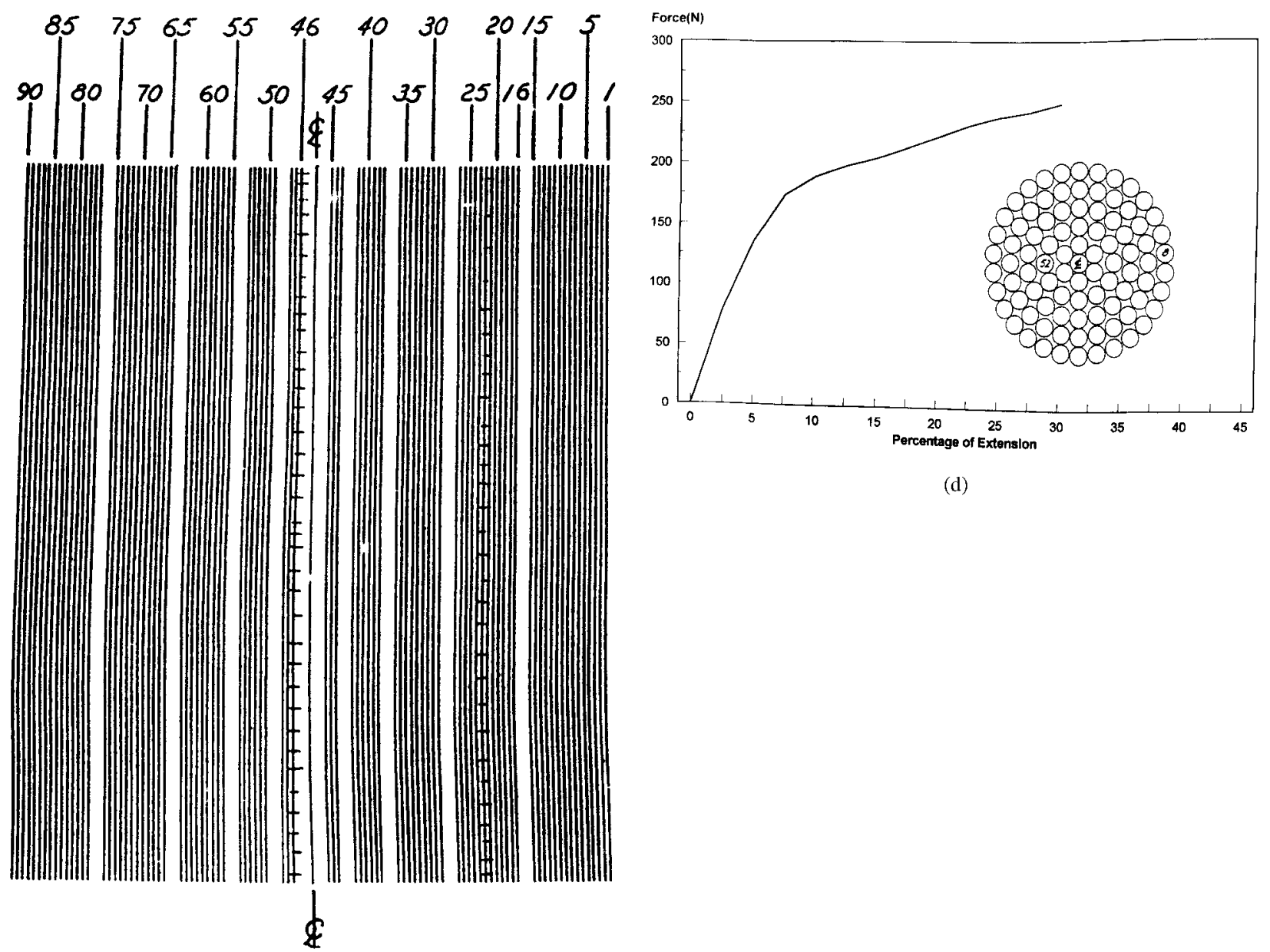

(d)

(c)

Figure 2 (Continued from the previous page)

tween the blended fiber types on the yarn properties are investigated.

\section{The Mechanics of Blended Yarns with the Statistical Variations}

In view of the stochastic nature of material strength, statistical approaches have been applied as well. Phoenix ${ }^{57,96-98}$ proposed the concept of the chain-of-bundles model of the strength of yarns to tackle the issue of statistical nature of strengths of individual filaments and yarns, the size (length) effect on filament strength, as well as the load-sharing mechanism during yarn breakage.

Meanwhile, extensive experimental and theoretical work has been done by Monego et al. ${ }^{45,99,100}$ using mechanical tracer elements as a means of studying rupture mechanisms in continuous twisted structures of blended fiber types.
Also recently, Pan and Postle ${ }^{51}$ have completed an analysis on strength prediction of blended yarn structure by including the fiber property variations and the interfiber interactions. Hybrid structures have been applied in many areas such as textiles and composites. However, the mechanisms giving rise to the advantages associated with fiber blending, presumably because of the interactions between constituents of different types, are still poorly understood. This work is an attempt to look into the internal interactions through theoretical analysis. A twisted structure (yarn) of two distinct fiber types with statistically distributed strengths is studied. It has been established in yarns of single-fiber type ${ }^{27}$ that the interaction via friction between fibers will lead to a substantial increase of fiber effectiveness, and consequently the yarn strength. Incorporating this concept into the hybrid case makes it possible 


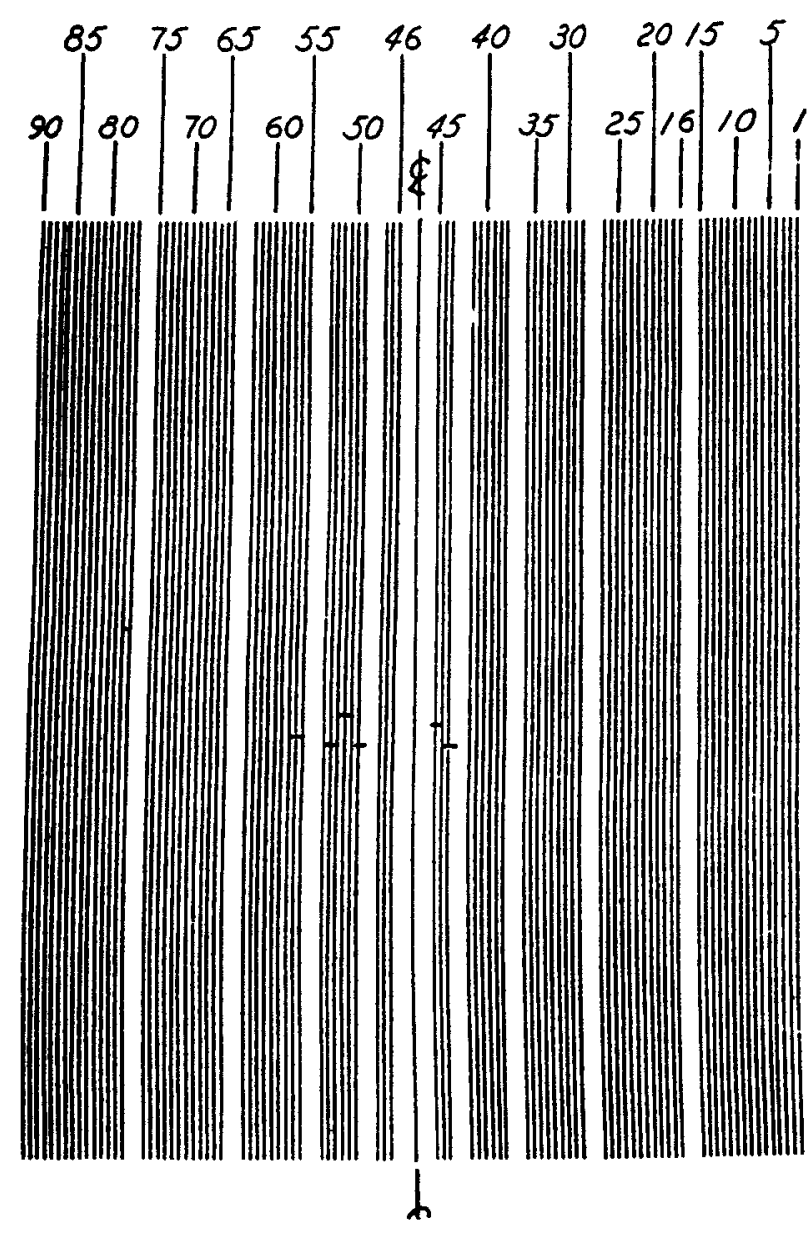

(a)

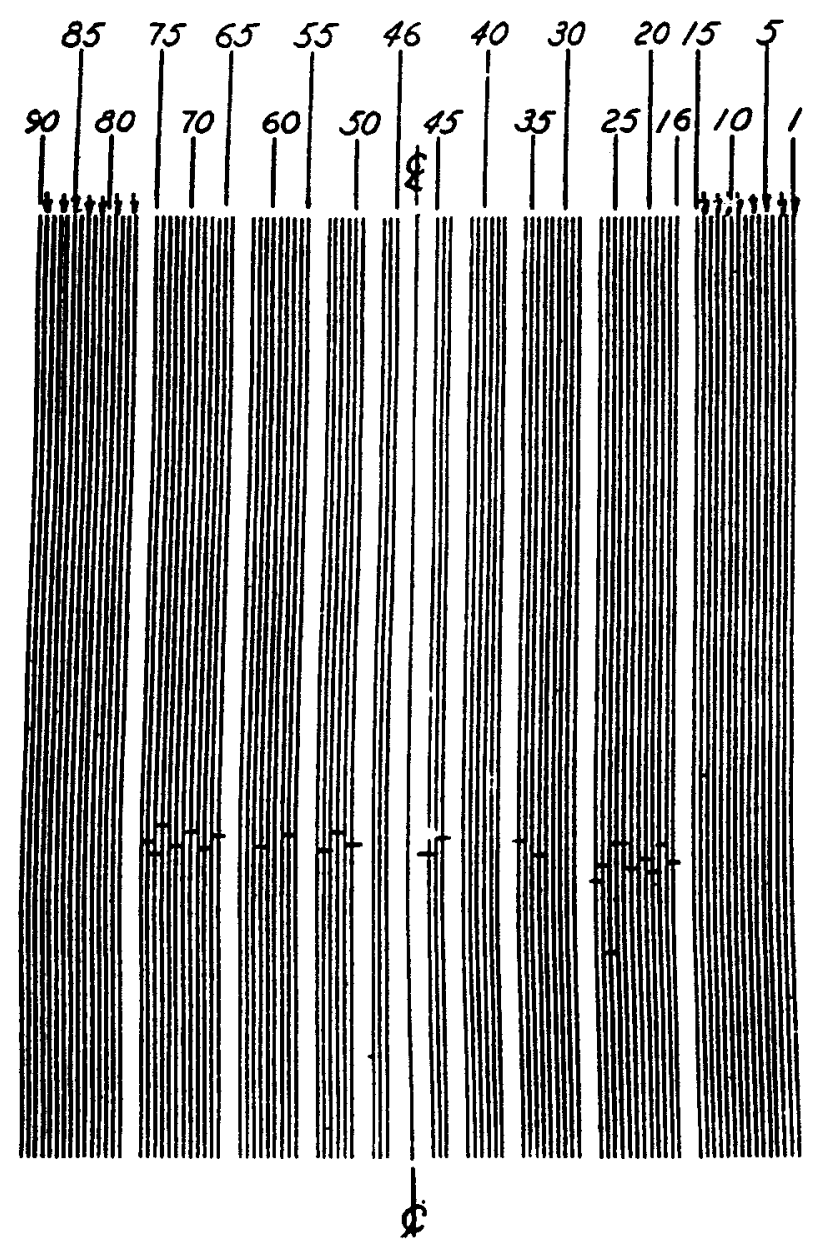

(b)

Figure 3 Experiments of a model with 40 cotton and 51 dacron components at TM $=3.26^{99}$ : (a) multiple breaks at $11 \%$ extension; (b) multiple breaks at $12 \%$ extension; (c) multiple breaks at $13 \%$ extension; and (d) yarn cross-section and its load-extension curve.

to more accurately predict the strength and its distribution for the blended yarn.

\section{Size or Scale Problems}

The gauge length at which a material is tested for its mechanical behavior has long been recognized as an important factor influencing such properties as strength and extensibility. Recent research $^{101,102}$ has revealed more information about the importance of specimen length. Contrary to a common assumption that the initial modulus of a fiber is independent of its length, it was found that the initial modulus decreases as the gauge length is reduced, because the test length affects the strength and breaking strain of the product by different amounts. Also, it was observed that the impact of gauge length on mechanical properties differs between single fibers and fibrous structures such as yarn or fabric. In a fibrous structure, fragmentation occurs during tensioning of the structure and greatly diminishes the gauge length effect, leading to a much weaker connection between the mechanical properties and the specimen length.

Further, in all previous analyses, the critical length in the fragmentation process used for calculating system strength is the value derived under a normally implicit assumption that the fragmentation process has reached the saturated stage. As a result, for a given twisted structure, this critical length will be constant, and the strength of the system thus becomes length-independent. It was found that, in the real cases, 

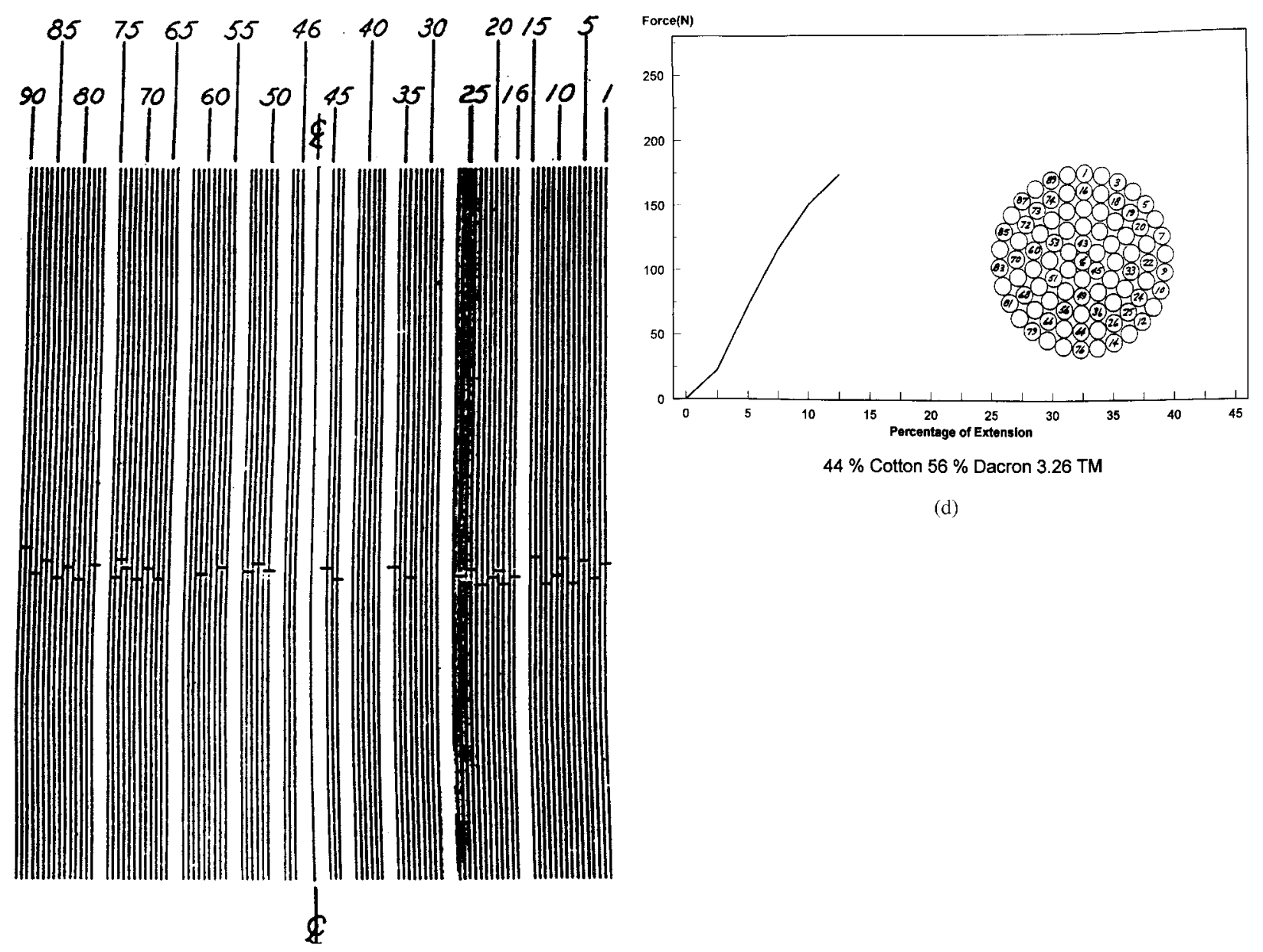

(d)

Figure 3 (Continued from the previous page)

there are many variables that affect the fragmentation process adversely so that a saturated fragmentation state is often not realized, hence resulting in the strength-length dependence experimentally observed in a fibrous structure.

\section{EXPERIMENTAL EVIDENCE AND ISSUES}

The progresses summarized above are either developed owing to the inspiration from, or verified by, the experimental results shown below.

\section{The Fragmentation and the Critical Fiber Length}

Introduced here is an early experimental work that delivers informative and elaborate experimental evidence obtained under the conditions close to ideal, and hence reveals some fundamen- tal confirmations supporting our discussions above.

This work was done by Monego et al., ${ }^{45,99,100}$ using mechanical tracer elements as a means of studying rupture mechanisms in continuoustwisted structures of blended-fiber types. To facilitate experimental control and observation, they made a set of gross-model yarns in lieu of conventional structures. The gross-model yarns each consisted of 91 components, either cotton yarns or polyester (termed PET or Dacron below) filament yarns, drawn from independent packages in a creel and twisted carefully with negligible radial migration, in five helical layers about a central or core yarn. A total of 91 different colors was used for different samples of the cotton yarn to permit identification of each cotton component with respect to its radial position in the gross model. A range of such model structures were prepared 
which varied from 0 to $100 \%$ cotton (100 to $0 \%$ polyester) with twist ranging from about 0.50 to 4.50 twist multiplier.

In this model yarn (or more accurately, a strand consisting of individual yarns or components), the polyester components are yarns of 70 denier, formed by 34 filaments, and the cotton yarns are of 79's cotton count or 67 denier. For briefness, we will refer to this model strand as a blended yarn, and its constituent yarns as components.

Successive specimens of these model strands were tested on an Instron tester to different strain levels. After each specimen was strained to its designated level, it was removed from the instrument, then carefully untwisted and examined for the presence of tensile breaks in the component yarns. A fairly simple approach was used to record the location and frequency of component breaks at each extension level for each gross model. A series of parallel lines was drawn in groups corresponding to half the number of components in each ring of the model cross-section. Each group was then arranged symmetrically about the center line or axis of the model. The lines were numbered arbitrarily from right to left as shown in Figure 2. Thus, each numbered line corresponds to a numbered position in the crosssection of the model in Figure 2(d). The unmarked locations represent polyester yarn positions for the given specimen. A few testing results are provided herein as a basis for further discussion.

Figure 2 shows data taken in tests of a 2-cotton/89-polyester component model with the cotton located at positions no. 8 and no. 52, as shown in Figure 2(d). The twist multiplier was 2.19. After extension of the model yarn to the $11 \%$ strain level, component no. 52 , located in the second ring from the core, evidenced five breaks in its

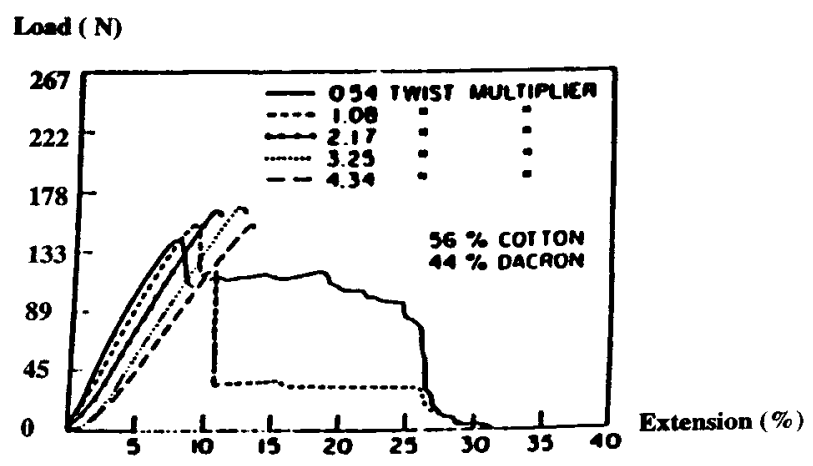

Figure 4 Effects of twist level on fracture behavior of yarn models (experimental ${ }^{99}$ ). 20.32-cm gauge length (this process of multiple breaks is now widely termed as the fragmentation process), whereas component no. 8 in the fifth or outer ring showed no breaks, as seen in Figure 2(a). When another specimen of the same model was extended to $15 \%$, it evidenced 19 breaks in no. 52 and fewer breaks in no. 8, as shown in Figure 2(b). Figure 2(c), representing the effect of $25 \%$ extension, shows the presence of 44 breaks in no. 52 and 13 in no. 8.

As mechanical tracers, components no. 52 and no. 8 clearly illustrate the dependence of multiple break frequency on location, hence on local strain level and on local pressure. The whole structure will fail when the length of the fragment reaches the critical length and can no longer build up load.

In contrast, an entirely different fracture behavior can be observed by using a 3.26 twist multiplier model of 40 cotton components. The initiation of rupture of a few inner cotton components is followed by rapid and concentrated propagation of the break to most of the cotton yarns in that region. At this point, the load shed so precipitously by the cotton exceeds the additional loadbearing capacity of the polyester and the entire model fails in a manner characteristic of a $100 \%$ cotton model. The remaining elongation of the polyester is not realized, as is seen in Figure 3. The concentration of the break propagation is seen in Figure 3(a-c) for extension steps of 11, 12, and $13 \%$, respectively.

Finally, it is shown in Figure 4 that twist strongly influences the load transfer and rupture propagation and thus, by altering the intercomponent interactions, significantly affects the level of tensile strength (or the in situ component behavior), the reaching of (or failure to reach) the full filament extension potential (the saturation of the fragmentation process), the overall shape of the yarn stress strain curve and its general slope and, eventually, the work to tensile rupture. In these tests from Figure 4, all models were the same blend ratio, 56-cotton/44-polyester. The various twist levels ranging from 0.54 to 4.34 twist multiplier lead to quite diverse fracture responses.

The significance of this work includes:

1. It clearly demonstrates the distributions of the fiber strain and the lateral pressure in a yarn

2. It stresses the importance of the intercomponent interactions in a fibrous structure 
3. It provides for the first time the experimental evidence of the fragmentation process and the critical fiber length in a twisted fibrous structure

4. It shows that the saturation of the fragmentation process is not always realized; therefore, yarns at different situations will break with different critical length, leading to the gauge length effect in the yarns

5. It illustrates the existence of the difference between the in situ and ex situ fiber behaviors.

6. Finally, these experiments verify the existence of the hybrid effect in a blended structure. There are normally two peaks in the stress-strain curve of a blended yarn; the first one is associated with the cotton component and the second one with the PET component. By examining Figure 4, we can see that the locations (breaking strains) of the two peaks shift depending on the twist level. In other words, in this blended case, the two components behave differently at different twist levels, and from what they would in a nonblended case.

\section{The Hybrid Effects and the Fiber Property}

There are further experimental verifications for the hybrid effect. Kemp and Owen ${ }^{103}$ studied experimentally the strength and mechanical behavior of nylon/cotton blended yarns, and they found that there exists a dependence between the behavior of two fiber types: the cotton fibers in the blended yarn break at strains considerably less than the breaking strain in an all-cotton yarn.

$\mathrm{Pan}^{104}$ also performed a related experiment by using two sets of yarns. The first set of yarn samples were made of polyamide 66 (nylon-66) and polypropylene filaments. By altering the numbers of nylon-66 and polypropylene filaments, the blend ratio of each yarn was adjusted. The yarns were twisted to different degrees according to the experiment design. The yarn samples were tested on an Instron tester.

The second set of data are from Monego ${ }^{99}$ used before. It is thus possible to construct Figure $5(\mathrm{a}, \mathrm{b})$ in terms of tenacity versus the blend volume fraction $W_{1}$. For the first set of data, the nylon-66 fiber was chosen as the reinforcing fiber in which volume fraction is therefore designated as $W_{1}$. For the second set, the PET component is the reinforcement. There are two results in each

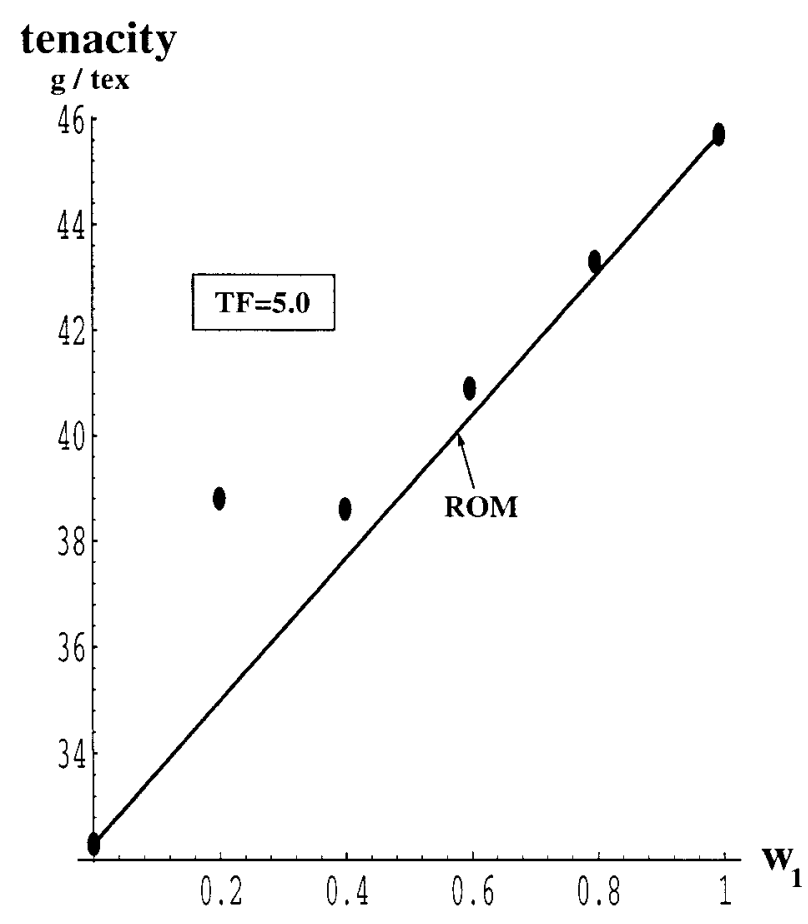

(a)

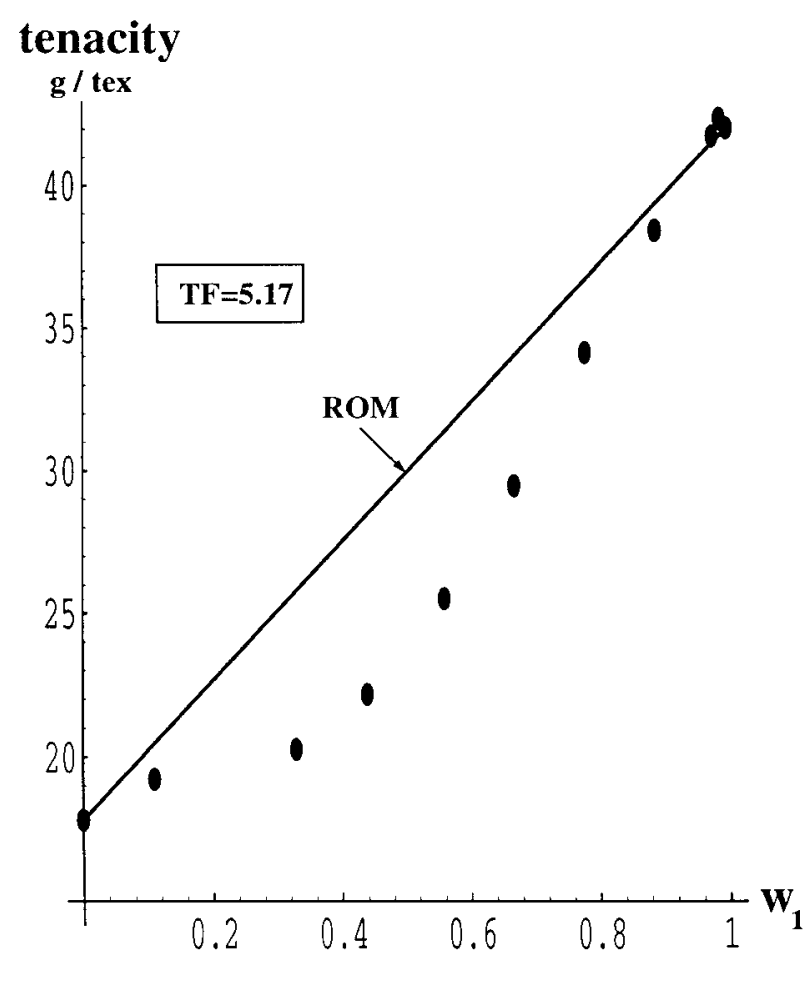

(b)

Figure 5 The tenacity versus the blend ratio $W_{1}$ and the comparison between the prediction by the rule of mixtures and the experiments: (a) for the first set of yarns; (b) for the second set of yarns. 
figure, the line representing the prediction based on the rule of mixtures (ROM) and the points of the experimental results. So the difference between the results of ROM and the experiments shows the existence of the hybrid effect, according to the definition associated with ROM.

As seen in the figures, for the two sets of yarns tested in this study, they have demonstrated different behaviors in terms of hybrid effects. For the first group, the hybrid effects are much smaller than those for the second group. To explain the differences, we can turn to a previous publication. ${ }^{27}$ In that article, it was predicted that increasing tensile modulus ratio $E_{f 1} / E_{f 2}$ of the two fiber types in a blended yarn will lead to a more significant hybrid effect. When we checked the fiber data, we found that the ratio $E_{f 1} / E_{f 2}$ $=1.09$ for the first set of yarn is lower than $E_{f 1} / E_{f 2}=2.71$ for the second set. This accounts for the difference in the hybrid effect between the two groups of yarns.

Just recently, Pan et al. ${ }^{105}$ have published an article that verifies more thoroughly the existence of the hybrid effects and the involved variables for hybrid fibrous twisted structures through a systematic experiment.

\section{The Size Effect at Different Levels of Fibrous Structures}

Pan et al. ${ }^{102}$ have performed another experimental work to examine the size effect at different levels of fibrous structures with a series of tensile tests on single fibers, fiber bundles, and yarns. Three types of filaments, polypropylene, polyester, and nylon-66 were selected for the project. Details of the results can be found in Pan et al. ${ }^{102}$ Figure 6 shows the gauge length effect on the relative strength for polypropylene, including single fiber, fiber bundle, and twisted yarns. It is clearly shown that fiber has the most significant gauge length effect, the gauge length influence reduces for bundle probably because of the fiberfiber interactions, and diminishes further for the yarn because of the fragmentation process.

\section{CONCLUSIONS}

Part of the fibrous structure manufacturing industry associated with clothing applications has been considered a "sunset" or a mature industry at least in the developed countries. However, if we look at the current state of textile science, we

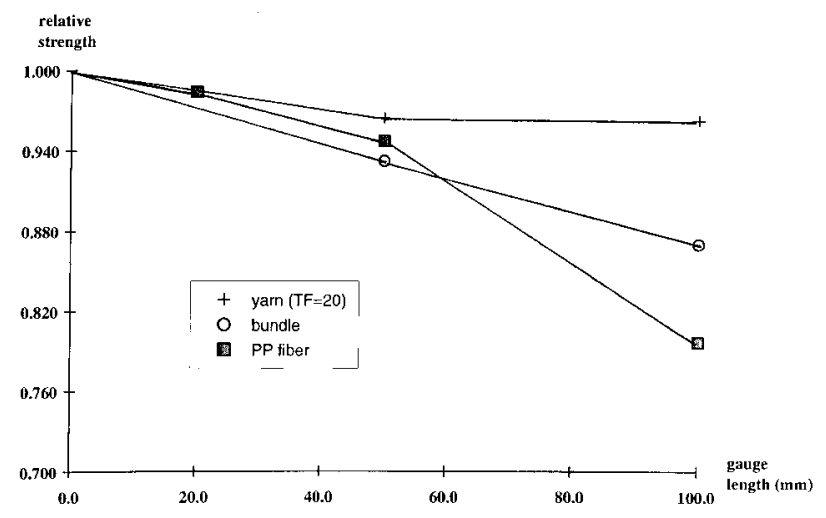

Figure 6 The gauge-length effect on single fiber, fiber bundle, and yarn.

have to admit that, despite the extensive progress we have made, compared with other fields, textile science is still in its adolescence, and very much underdeveloped, due partly to the extreme complexity of textile structure itself, and partly to the almost negligence in the past by the scientific community at large-no science discipline is able to grow in isolation. There are many fundamental issues and problems yet to be solved in order to establish a knowledge system mature enough or fully developed to support and guide the advance of the industry.

Since the 1990s, we have witnessed an arousing interest in research of textile or fibrous structures and expanding applications of the materials. There are several reasons likely attributed to this change. First, textile-reinforced composites and other textile structures for load-carrying industrial applications provide some unique advantages that are nonexistent in the traditional engineering materials, and therefore attract attention from various engineering fields; second, with the ending of the "Cold War," defense-related research expenditure has been severly reduced, and part of the savings is shifting, as a peace dividend, toward research for consumer products such as textiles. Although no one knows for sure whether this shifting indicates a long-term change or just a transitional incident, it is certainly a welcome sign for the textile industry.

The authors thank Prof. Stanley Backer of MIT for many inspiring and helpful discussions during the preparation of the manuscript.

\section{REFERENCES}

1. The Textile Institute. Textile Terms and Definitions, 9th ed.; 1991. 
2. Messe Frankfurt. Techtextil: Hightex for a Better Living, 1991.

3. Wilson, K. A History of Textiles; Westview Press: Boulder, CO, 1979.

4. Backer, S. in Mechanics of Pneumatic Tires; Clark, S., Ed.; Monograph 122; National Bureau of Standardization; Washington, D.C., 1971.

5. Hearle, J. W. S.; Grosberg, P.; Backer, S. Structural Mechanics of Yarns and Fabrics; Wiley-Interscience: New York, 1969; Vol. 1.

6. Hearle, J. W. S.; Thwaites, J. J.; Amirbayat, J. Mechanics of Flexible Fiber Assemblies; Sijthoff \& Noordhoff: Alphen an den Rijin, The Netherlands, 1980.

7. Kaswell, E. R. Textile Fibers, Yarns and Fabrics; Reinhold: NY, 1953.

8. Postle, R.; Carnaby, G.; de Jong, S. The Mechanics of Wool Structures; Ellis Horwood: New York, 1988.

9. Parsey, M. R. in Proceedings of the Offshore Technology Conference, Houston, TX, Paper 4307, 1982.

10. Hsu, P. Ph.D. Thesis, Massachusetts Institute of Technology, Cambridge, MA, 1984.

11. The Cordage Group. In Rope Technical Data; Columbian Rope Co.: Auburn, NY, 1977.

12. Hearle, J. W. S. Text Horizons 1996, 16(2), 12.

13. Wu, H. C.; Seo, M. H.; Backer, S. Text Res J 1995, 65,619 .

14. Seo, M.; Mandell, J. F.; Backer, S. in Proceedings of the Marine Technology Society Conference; Washington, DC, 1990.

15. Gegauff, G. Bull Soc Ind Mulhouse 1907, 77, 153.

16. Platt, M. M. Text Res J 1950, 20, 1.

17. Hearle, J. W. S. J Text Inst 1958, 49, T389.

18. Treloar, L. R. G. Br J Appl Phys 1962, 13, 314.

19. Kilby, W. F. J Text Inst 1964, 55, T589.

20. Wilson, N. Br J Appl Phys 1965, 16, 1889.

21. Costello, G. Theory of Wire Rope; Springer-Verlag: New York, 1990.

22. Pan, N.; Palmer, M. L.; Seo, M. H.; Boyce, M.; Backer, S. in Proceedings of the International Conference on Fiber and Textile Science, Ottawa, Canada, 1991; p 179.

23. Pan, N. Text Res J 1992, 62, 749.

24. Pan, N. Text Res J 1993, 63, 504.

25. Pan, N. Text Res J 1993, 63, 565.

26. Pan, N. J Text Inst 1996, 87, 467.

27. Pan, N. J Mater Sci 1993, 28, 6107.

28. Backer, S.; Seo, M. H. in Proceedings of the Third Japan-Australia Joint Symposium on Objective Measurement: Applications to Product Design and Process Control; Kawabata, S.; Postle, R. Eds.; Kyoto, Japan, 1985; p 653.

29. Backer, S.; Hsu, P. in Proceedings of the Third Japan-Australia Joint Symposium on Objective Measurement: Applications to Product Design and Process Control; Kawabata; Postle, Eds.; Kyoto, Japan, 1985; p 101.
30. Galilei, G. Dialogue Concerning Two New Sciences (Leyden, 1638); De Salvio, A.; Fabaro, A., Translators, Northwestern University Press: Evanston, IL, 1914.

31. Gurney, H. P. J Text Inst 1925, 16, 269.

32. Sullivan, R. R. J Appl Phys 1942, 13, 157.

33. Machida, K. M.S. Thesis, Massachusetts Institute of Technology, Cambridge, MA, 1963.

34. Grosberg, P. J Text Inst 1963, 54, T223.

35. Dogu, I. Text Res J 1972, 42, 726.

36. White, J. L.; Chen, C. C.; Spruiell, J. E. Appl Polym Symp 1975, 27, 275.

37. Backer, S. Text Res J 1952, 22, 668.

38. Zorowski, C. F.; Chen, C. S. Text Res J 1965, 35, 529.

39. Kim, S. Y.; Hersh, S. P.; El-Shiekh, A. Text Res J 1974, 44, 958.

40. Prakash, A.; Conaway, T.; Costello, G. J Appl Mech 1992, 59, S213.

41. Coplan, M. J. in Blend Fabrics and Their Impact on Military Textile Applications; Proceedings of a conference, Quartermaster Research and Engineering Center, Natick, MA, May 17-18, 1960.

42. Gupta, D. K.; El-Shiekh, A. Appl Polym Symp 1975, 27, 295.

43. Hamburger, W. J. J Text Inst 1949, 40, 700.

44. Kelly, A.; Macmillan, N. H. Strong Solids, 3rd ed.; Clarendon Press: Oxford, 1986; p 252.

45. Monego, C. J.; Backer, S. Text Res J 1968, 38, 762.

46. Noshi, H.; Shimadu, M.; Kusano, T. J Text Mech Soc 1959, 42, 12, 91.

47. Ratman, T. V.; Shankaranarayana, K. S.; Underwood, C.; Govindarajulu, K. Text Res J 1968, 38, 360 .

48. Marom, G.; Fischer, S.; Tuler, F. R.; Wagner, H. D. J Mater Sci 1978, 13, 1419.

49. Bader, M. G.; Manders, P. W. J Mater Sci 1981, 16, 2233.

50. Harlow, D. G. Proc R Soc Lond 1983, A389, 67.

51. Pan, N.; Postle, R. J Text Inst 1995, 86, 559.

52. Pan, N.; Postle, R. Trans R Soc Lond A 1996, 354, 1875.

53. Peirce, F. T. J Text Inst 1926, 17, 355.

54. Daniels, H. E. Proc R Soc 1945, A183, 405.

55. Weibull, W. J Appl Mech 1951, 18, 293.

56. Harlow, D. G.; Phoenix, S. L. J Compos Mater 1978, 12, 195.

57. Phoenix, S. L. Text Res J 1979, 49, 407.

58. El-Mogahzy, Y. E.; Gupta, B. S. Text Res J 1993, 63, 219.

59. Gupta, B. S.; El-Mogahzy, Y. E. Text Res J 1991, $61,547$.

60. Kothari, V. K.; Sengupta, A. K.; Rengasamy, R. S.; Goswami, B. C. Text Res J 1994, 64, 519.

61. Rosen, B. M. Fiber Composite Materials; American Society for Metals: Metal Park, OH, 1965; p 37.

62. Huang, N. C. Text Res J 1978, 48, 61.

63. Huang, N. C. Int J Solids Struct 1978, 14, 579. 
64. Asvadi, S.; Postle R. Text Res J 1994, 64, 208.

65. Conway, T.; Costello, G. Int J Solids Struct 1952, 30, 553.

66. Conway, T.; Costello, G. J Eng Mech ASCE 1993, $119,833$.

67. Conway, T.; Costello, G. Int J Appl Mech 1993, 60, 534.

68. El-Shiekh, A. Text Res J 1974, 44, 343.

69. Vangheluwe, L.; Goswami, B. Text Res J 1998, 68, 150.

70. Anandjiwala, R. D.; Goswami, B. Text Res J 1993, $63,392$.

71. Anandjiwala, R. D.; Carmical, M.; Goswami, B. Text Res J 1995, 65, 131.

72. Seo, M. Doctoral Thesis, Massachusetts Institute of Technology, Cambridge, MA, 1988.

73. Axelrad, D. R. Micromechanics of Solids; Elsevier Scientific Publishing: New York, 1978.

74. Axelrad, D. R. in Continuum Models of Discrete Systems; Kroner, E.; Anthony, K. H., Eds.; University of Waterloo Press: Waterloo, Ontario, Canada, 1980; p 3.

75. Carnaby, G. A.; Pan, N. Text Res J 1989, 59, 275.

76. Lee, D. H.; Lee, J. K. in Objective Measurement: Applications to Product Design and Process Control; Kawabata, S.; Postle, R.; Niwa, M., Eds.; The Textile Machinery Society of Japan: Osaka, 1985; p 613.

77. Pan, N.; Chen, J.; Seo, M.; Backer, S. Text Res J 1997, 67, 907.

78. Perkins, R. W.; Ramasubramanian, M. K. in Mechanics of Cellulosic and Polymeric Materials; Perkins, R. W., Ed.; The American Society of Mechanical Engineering: New York, 1989; p 23.

79. van Wyk, C. M. J Text Inst 1946, 37, T282.

80. Komori, T.; Makishima, K. Text Res J 1977, 47, 13.

81. Komori, T.; Makishima, K. Text Res J 1978, 48, 309.
82. Komori, T.; Makishima, K. Text Res J 1979, 49, 550 .

83. Lee, D. H.; Carnaby, G. A.; Carr, A. J.; Moss, P. J. A review of Current Micromechanical Models of the Unit Fibrous Cell; WRONZ Communication, No. C113, 1990.

84. Pan, N. Text Res J 1993, 63, 336.

85. Komori, T.; Itoh, M. Text Res J 1994, 64, 317.

86. Pan, N.; Carnaby, G. A. Text Res J 1989, 59, 285.

87. Stewart, I. New Scientist 1992, Vol. 134, p 16.

88. Pan, N. Polym Compos 1993, 14, 85.

89. Cox, H. L. Br J Appl Phys 1952, 3, 72.

90. Nedderman, R. M. Statics and Kinematics of Granular Materials; Cambridge University Press: New York, 1992; p 21.

91. Grosberg, P.; Smith, P. A. J Text Inst 1966, 57, T15.

92. Pan, N. J Text Inst 1993, 84, 472.

93. Pan, N.; Yoon, M.-Y. Text Res J 1993, 63, 629.

94. Tsai, S. W.; Wu, E. J Compos Mater 1971, 5, 58.

95. Pan, N.; Yoon, M.-Y. Text Res J 1996, 66, 238.

96. Phoenix, S. L. Fiber Sci Technol 1974, 7, 15.

97. Phoenix, S. L. Int J Eng Sci 1975, 13, 287.

98. Pitt, R. E.; Phoenix, S. L. Text Res J 1981, 51, 408.

99. Monego, C. J. M.S. Thesis, Massachusetts Institute of Technology, Cambridge, MA, 1966.

100. Monego, C. J.; Backer, S.; Qui, Y. P.; Machida, K. Compos Sci Technol 1994, 50, 451.

101. Pan, N.; Chen, H. C.; Thompson, J.; Inglesby, M. K.; Khatua, S.; Zhang, X. S.; Zeronian, S. H. J Mater Sci 1997, 32, 2677.

102. Pan, N.; Chen, H. C.; Thompson, J.; Inglesby, M. K.; Zeronian, S. H. J Mater Sci 1998, 33, 2667.

103. Kemp, A.; Owen, J. D. J Text Inst 1955, 46, T-684.

104. Pan, N.; Chen, K. Z.; Monego, C.; Backer, S. Textile Res J 2000, 70, 502 .

105. Pan, N.; Chen, K. Z.; Monego, C. J.; Backer, S. Proc R Soc Lond 1998, 454, 1109. 